\title{
The model of keep temperature in bathtub
}

\author{
Yiyi Pan ${ }^{1, a}$ \\ ${ }^{1}$ North China Electric Power University, Baoding, Hebei Province, China \\ a591446429@qq.com
}

Keywords: Temperature Distribution, Heat Transfer, Thermodynamics.

\begin{abstract}
How to keep the water temperature even throughout the bathtub has attracted wide attention. In this paper, two models are established to analyze and discuss this issue. The entire work is composed of the analysis on water temperature distribution throughout the bathtub, the relation between water flow rate and temperature distribution and the effect of critical factors on water temperature.
\end{abstract}

\section{Introduction}

Background. As the acceleration of the pace of life and people' s working, bathing has been an increasingly important way to relax in our lives. It has a variety of functions for bathing with constant hot water temperature such as relieving stress, soothing emotion, cosmetology, slimming and improving sleep quality. Most people use the regular bathtub without a secondary heating system because of low price. Although this kind of bathtub only has the basic function of heat preservation, it still cannot meet the demand of keeping constant water temperature even throughout the bathtub. We are asked to build a model which keeps constant water temperature by controlling the amount of inflow water and the temperature of inflow water in the bathtub. Through the model, we can enjoy the constant water temperature with ordinary bathtub and a faucet.

\section{Symbol Description}

\begin{tabular}{cl}
\hline Symbol & Definition \\
$Q_{1}$ & Cooling water on the wall of the bathtub \\
$Q_{2}$ & Heat from the faucet \\
$Q_{3}$ & Heat dissipation of water surface \\
$Q_{4}$ & Endothermic of human \\
$\lambda_{1}$ & The thermal conductivity of bathtub \\
$\lambda_{2}$ & The thermal conductivity of Water \\
$\lambda_{3}$ & The thermal conductivity of the flesh \\
$T$ & The temperature we will keep in the bathtub \\
$T_{1}$ & Temperature of hot water at inlet \\
$T_{2}$ & The temperature of the bathroom \\
$T_{3}$ & Human body temperature \\
$\Delta T$ & Change of temperature in unit time \\
$\mathrm{t}$ & Time \\
$\delta_{1}$ & Bathtub thickness \\
$\delta_{3}$ & The width of human body \\
$\delta_{4}$ & Bubble thickness \\
\hline
\end{tabular}




\begin{tabular}{cl}
\hline$A_{1}$ & The area for Inner bathtub \\
$A_{2}$ & The area of faucet outlet \\
$A_{3}$ & The surface area of human body \\
$A_{4}$ & The surface area of bubble \\
$c$ & Specific heat capacity of water \\
$v$ & Faucet water flow rate \\
$b$ & Constant variable \\
$\alpha$ & Heat conversion factor \\
$P_{b}$ & Partial pressure of vapor for saturated air. \\
$P_{q}$ & The pressure of water vapour for ambient air \\
$m_{1}$ & Mass of water in bathtub \\
$m_{2}$ & Mass of water from faucet \\
$\rho$ & Density of water \\
$V$ & Maximum water capacity in bathtub
\end{tabular}

\section{The Model}

Model : Thermodynamic model. In our daily life, around us there is heat transfer, when the water temperature in the bathtub is higher than that in the surrounding environment, the water will reduce the heat dissipation and temperature. At this time in order to ensure that the water in the bathtub to maintain constant temperature, we need to calculate the water heat loss by establishing a thermodynamic model, thus to know how much heat we should add to keep it constant. But the heat of the water in the bathtub through a variety of ways to dissipate, such as radiation of water surface, wall heat transfer and heat loss by people. But different heat loss have different calculation formula. So we need to establish a thermodynamic model of a variety of heat transfer equations to solve the relationship between temperature and time and heat flow, then calculate the best strategy to maintain the water temperature in the bathtub.

Establishment of Model. The bathtub wall heat loss. As long as there is a difference in temperature, the heat will spontaneously from the high-temperature objects to the low-temperature objects. is the heat loss due to thermal conduction of heat by the water in the bathtub which is in contact with the wall of the bathtub. It associated with the thermal conductivity of the bath tub, water and bath wall contact area, the thickness of the bath and the temperature difference between the two surfaces of the bathtub.

So the formula is

$$
Q_{1}=\lambda_{1} A_{1} \frac{T-T_{2}}{\delta_{1}}
$$

We can know the bathroom temperature is the function of ' $\mathrm{t}$ ' by the knowledge of non-steady heat transfer :

$$
T_{2}=299.15+298.15 e^{-t}
$$

$Q_{2}$ represents the heat supplied by the water tap. The water in the bathtub is cooled to a certain temperature by the water flowing out of the tap. So tap water provides heat for the water in the bathtub and the calculation formula of heat of water gives heat supplied by heat source:

$$
Q_{2}=c m_{2}\left(T_{1}-T\right)
$$


The mass of the water flowing into the bathtub is not a constant, it is a function of the flow rate of water, and can be derived from the continuity equation of fluid mechanics.

$$
m_{2}=\rho v A_{2}
$$

In order not to cause unnecessary waste, the water flow rate is not a constant, it will increase with room temperature and reduce, thereby reducing unnecessary waste, can achieve the best strategy by adjusting the constant $b$

$$
v=b / T_{2}
$$

$Q_{3}$ is an empirical formula, it is a total heat emitted from water surface, $\alpha$ is coefficient of heat transfer, $v_{a}$ is air flow rate, it includes the water convection heat, radiation heat and evaporative heat:

$$
Q_{3}=\alpha(0.089+0.4078 v \alpha) \frac{\Delta \mathrm{P}}{Y}
$$

$Q_{4}$ represents the endothermic formula, when people are in the bath, because the temperature of the water in the bathtub is higher than his body temperature, the body will absorb water heat, of course, body temperature will rise:

$$
Q_{4}=\lambda_{3} A_{3} \frac{T-T_{3}}{\delta_{3}}
$$

This is an empirical formula for the surface area of a person. It is related to the height and weight of a person:

$$
A_{3}=0.0061 h+0.0128 w-0.1592
$$

The temperature of the person in the bathtub can't always rise, it could only rise to the same temperature as the water in the bathtub, so on the basis of the knowledge of non steady heat transfer can be obtained.:

$$
T_{3}=310.15+3 e^{-t}
$$

As we finally have to keep the temperature of the water in the bathtub to be identical, the amount of water flowing into the bathtub is equal to the amount of water flowing out., so within the same time $\Delta t$, the heat of the bath tub is conserved, the loss of heat should be equal to the added value of the heat, based on such a relationship, we can get a first order differential equation of $\mathrm{T}$ on $\mathrm{t}$, we only need to solve the solution of this differential equation, we can get the temperature of the water bath temperature on the relationship between time and flow:

$$
c m_{1} \Delta T+Q_{1} \Delta t+Q_{3} \Delta t+Q_{4} \Delta t=Q_{2} \Delta t
$$

$m_{1}$ the weight of the water in the bathtub should be a constant value

$$
m_{1}=\rho V
$$

Finally, finishing above formulas, we can obtain the following formula.

$$
c m_{1} \frac{\Delta T}{\Delta t}=\lambda_{1} A_{1} \frac{T_{2}}{\delta_{1}}-\left(\frac{\lambda_{1} A_{1}}{\delta_{1}}+\frac{\lambda_{3} A_{3}}{\delta_{3}}+c \rho v A_{2}\right) T+c \rho v A_{2} T_{1}+\frac{\lambda_{3} A_{3}}{\delta_{3}} T_{3}+\alpha\left(0.089+0.4078 v_{a}\right) \frac{\Delta \mathrm{P}}{Y}
$$

Model solving

Simplify above model

$$
A \frac{\Delta T}{\Delta t}=B T_{2}-(B+C v+D) T+C v T_{1}+D T_{3}+E
$$

Bring the data we collected into the above equation, we can find out the value of these five coefficients: 


$$
\begin{aligned}
& \boldsymbol{A}=9.177295 \times 10^{2} \\
& \boldsymbol{B}=16.5775335 \times 10^{2} \\
& \boldsymbol{C}=5.254 \\
& \boldsymbol{D}=3.6865 \\
& \boldsymbol{E}=44.25510 \times 10^{2}
\end{aligned}
$$

\section{Result and Analysis}

We need to use the mathematical model established above to solve the optimal solution, the problem of solving the mathematical model above can be translated into the problem of solving the optimal flow velocity function. Therefore, we need to follow a fixed process and repeated regulating velocity function. Only do this, we can obtain the optimal flow velocity function. So we could obtain the best strategy.

The primary parameter is the maximum temperature in environment, when we analyze the sensitivity of the temperature of human. We observed the result of the model by changing the limit of the maximum temperature.

Equally, the primary parameters are the surface area and the volume of human body when we analyze the sensitivity of the shape and volume of human. Therefore, we choose child to test sensitivity which could distinguish evidently.

The primary parameters are the volume of the bathtub and the mass of the water when we analyze the sensitivity of the bathtub volume. Control other variables unchanged but increase the volume of the bathtub. The following is the changes of the model results.

\section{References}

[1] Asdrubali F. A scale model to evaluate water evaporation from indoor swimming pools[J]. Energy and buildings, 2009, 41(3): 311-319.

[2] Maximum heating temperature of water heater http://baike.baidu.com/link?url=UjcBNREkHj8 xfEhEH-XsXwi9RXcMkJU0ZPkz_q_nDr4jnB-F7d2Am8Uv_SIXXfXRcDdhHvQODlhE-o2EC7jej K\#1_1

[3] The most suitable temperature of bath http://szb.dlxww.com/xsb/html/2010- 05/10/content_ 350609.htm

[4] The model of the Constant temperature box http://wenku.baidu.com/link?url=WdrqT5zk6zYtCg gstQPeRp1v6v6ezAOGnJRTI419MLYS2vMJuPauJX61o2t2pT2WKV18o6cwzhTgzpUpLUXnVm GT4gq9katXd6BusXcH67

\section{[5]Heat Transfer}

[6] Coefficient of thermal conductivity of water http://zhidao.baidu.com/link?url=kYYCaCqPym0q h9NjgMULP1uhix4HSB_Pj81YIKjatmZuQ3JcfSgEVIh46Iz1G1y1RW4q22g8xRp8baXaxdDK_\& qq-pf-to=pcqq.group

[7] Coefficient of thermal conductivity of human http://www.zybang.com/question/dc7d89508a d361f86c5ed8e74ccc8628.html 